\title{
Wavelet Packet Energy Feature Extraction Method of Recoil Mechanism Wear Signal
}

\author{
LUO Jianhua ${ }^{1, ~ a ~}$, LI Hua $^{1, ~ a ~}$, Zhong Mengchun ${ }^{1, ~ a ~}$, ZHANG Chunlin $^{2, \text { b }}$
}

${ }^{1}$ Department of Arms Engineering, Academy of Armored Force Engineering, Beijing, 100072, China

${ }^{2}$ Jiangnan computer research, Wuxi, 214000, China

aemail: iuanne@163.com, b dspcan@163.com

Keywords: wavelet packet; energy feature; feature extraction; forecast

\begin{abstract}
According to the characteristic of instantaneous, non-stationary and non-linear of the vibration signal, wavelet packet energy feature extraction is proposed, and the theoretical foundation of this method and concrete implementation method is explained in detail. Finally, the relations of characteristic quantity of recoil mechanism and the wear of control loop on the basis of feature extraction methods based on wavelet packet energy is established.
\end{abstract}

\section{Introduction}

Anti-Recoil equipment is different from the general mechanical equipment. The integrity of the anti-recoil's states is an important guarantee for firing so that is an important part of tank weapon system. There are certain faults of anti-recoil such as air leakage, liquid leakage (liquid amount is less) as well as worn of control billots, control ring. Many common faults could be excluded in advance before the tank gun firing through service inspection, however, there is one fault that couldn't be excluded is worn failure. Therefore, this paper focusing on study the changes of feature quantity caused by the worn of control billots, control rings.

\section{Study of Anti-Recoil Equipment Worn State Feature Extraction Method}

When anti-recoil wear failure occurs, the amplitude and phase characteristics of different signal segments in different frequency will be changed into different degree. When a signal which containing a different frequency components incent on the anti-recoil system will be inhibited or enhanced because of the worn fault that has positive or negative effect on certain frequency. Therefore, the energy of different frequency band will be changed. If the fault played the role of inhibition to the frequency, the energy is reducing. Otherwise, the signal energy of certain frequency band is increasing. So it's easy to establish an "Energy - fault" fault diagnostic model. This method is discriminating the fault signal by which portion of the frequency characteristics changes. This establishes a correspondence between the frequency change and the failure, and characterization of frequency used by the feature quantity is the energy[1].

On such issues, reference [2] using wavelet multi-resolution analysis to solve. Wavelet packet decompose can divide $L^{2}(R)$ 's subspace $W_{j}$ into $2^{k}$ orthogonal subspace's summation. Using wavelet packet further dividing the ${ }^{W_{j}}$ into $2^{k}$ mutually independent frequency sub-bands in case of remaining unchanged time scale and subspace level. The method greatly improved the frequency localized capacity and resolution ratio of time domain analysis, furthermore, overcome the defects of wavelet multi-resolution analysis [3].

The signal through orthogonal wavelet packet decomposition could be divided into various frequency that each band are mutually independent. So we can use wavelet packet method for monitoring the various signal components. In practical, the wavelet packet signal amplitude square summation can be used to indicate different frequency of the signal segment energy rate [4]. 


\section{The Principle of Packet Energy Feature Extraction Method}

$X_{i j}$ is the $i$ layer and $j$ node coefficient of vibration signal $S$ which has been noise reduction and wavelet packet decomposed. Reconstructed the Coefficients that obtained by the wavelet packet could extracted every frequency band time-domain signal. Using the $S_{i j}$ to represent the reconstructed signal, and the original signal could be expressed as:

$$
S=\sum_{j} S_{i j}
$$

In the formula, $i$ represents wavelet packet decomposition level, and $j$ is the node number. Then $E_{i j}$ which represents the energy of $S_{i j}$ could be expressed as:

$$
E_{i j}=\int\left|S_{i j}(t)\right|^{2} d t=\sum_{j=1}^{n}\left|X_{i j}\right|^{2}
$$

In the formula, $x_{i j}(j=1,2, \ldots, n)$ shows the signal $S_{i j}$ amplitude of each discrete points. Thus, feature vectors can be constructed as follows:

$$
T=\left[E_{i 1}, E_{i 2}, E_{i 3}, \ldots, E_{i 2^{i}}\right]
$$

In practical engineering of signal analysis, the energy is usually a great value. Therefore, we need to normalize the eigenvector $T$. So as follows:

$$
\begin{aligned}
E & =\sum_{j} E_{i j} \\
T^{\prime} & =T / E
\end{aligned}
$$

The vector $T^{\prime}$ is the normalized eigenvector.

When the signal being wavelet packet decomposing, the decomposition level should be choose that depending on the signal characteristics and requirements of the characteristic parameters. On the one hand, too small decomposition level will lead to fuzzy result. On the other hand, too much decomposition levels will course the increase of feature vectors' dimension which decrease the computation speed.

\section{The Feature Extraction of Vibration Signal's Wavelet Packet Energy}

Collect the data of acceleration signal of anti-recoil equipment in six rounds live fire test and pretreated that filter out the low frequency errors due to charge drift and noise signal bring by collector, sensor via electromagnetic environment. Comparing and observing 6 rounds vibration signal's performance in the time domain and frequency domain, as shown in Figure 1-6.

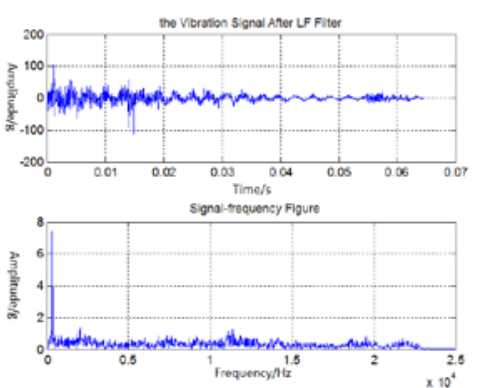

Fig. 1 the 1st Shell Vibration Signal Time-frequency Figure

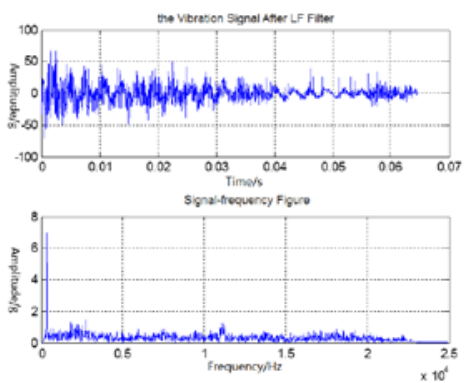

Fig. 2 the 2nd Shell Vibration Signal Time-frequency Figure

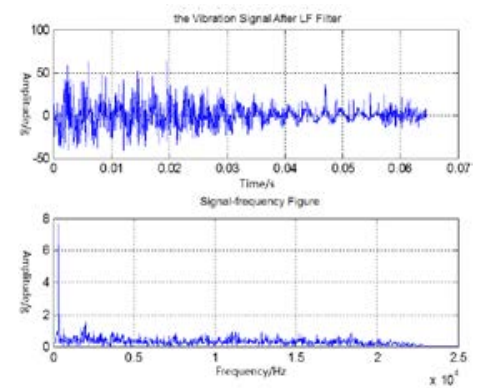

Fig. 3 the 3rd Shell Vibration Signal Time-frequency Figure 


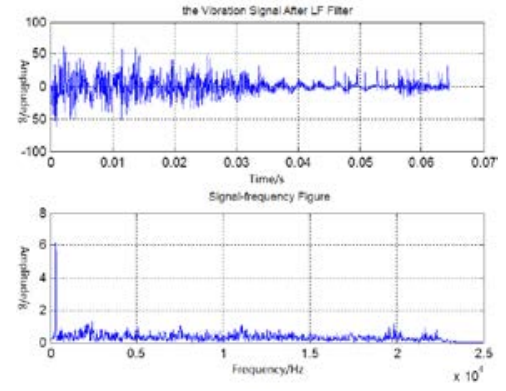

Fig. 4 the 4 th Shell

Vibration Signal

Time-frequency Figure
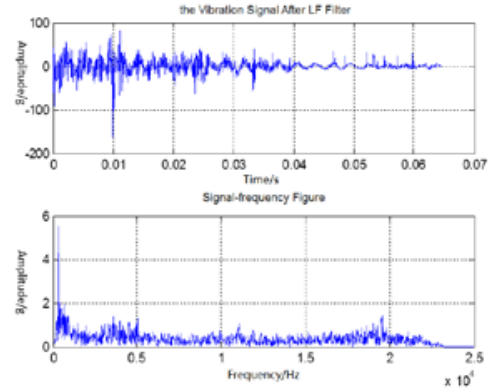

Fig. 5 the 5th Shell

Vibration Signal

Time-frequency Figure
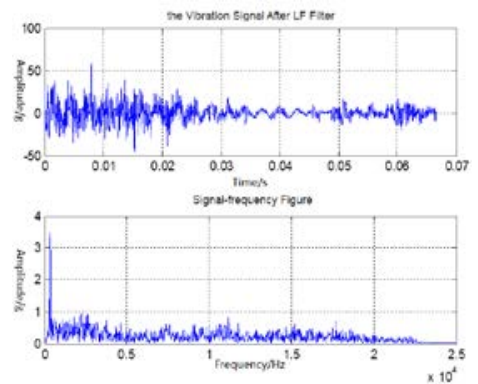

Fig. 6 the 6th Shell Vibration Signal Time-frequency Figure

By comparing and observing the waveforms of vibration signal in the frequency domain figure, we could found the energy which course by the recoil progress mainly in $338 \mathrm{~Hz}, 2000 \mathrm{~Hz}, 7500 \mathrm{~Hz}$, $11100 \mathrm{~Hz}, 19500 \mathrm{~Hz}, 22000 \mathrm{~Hz}$ frequency around. Therefore, utilizing the above-mentioned extraction method based on energy wavelet packet to decompose the signal into 4 layers wavelet packet. Choosing 'db5' wavelet type and 'Shannon' entropy. Reconstruct six frequencies band after decomposition and shown the results in Figure 7-12. The decomposed signal are again solving energy of each band, then constitute them to anti-recoil equipment feature vectors, as shown in Table 1.
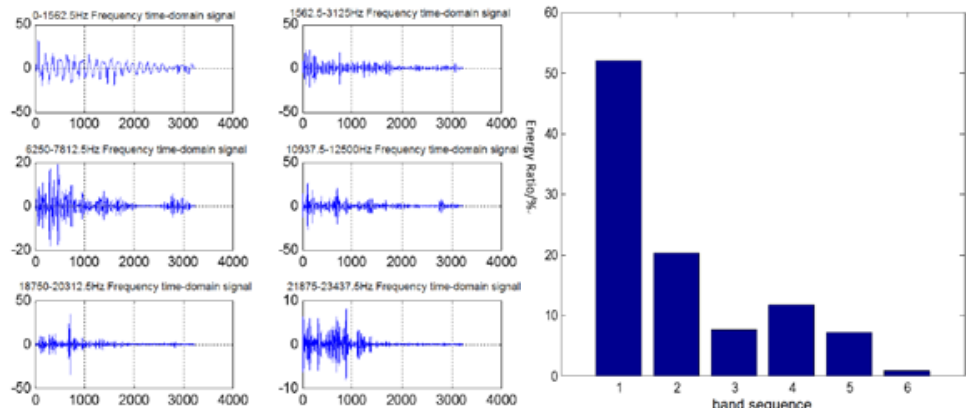

Fig. 7 the 1st Shell Recoil Progress Vibration Signal each Band Time-domain and Energy

Distribution Diagram
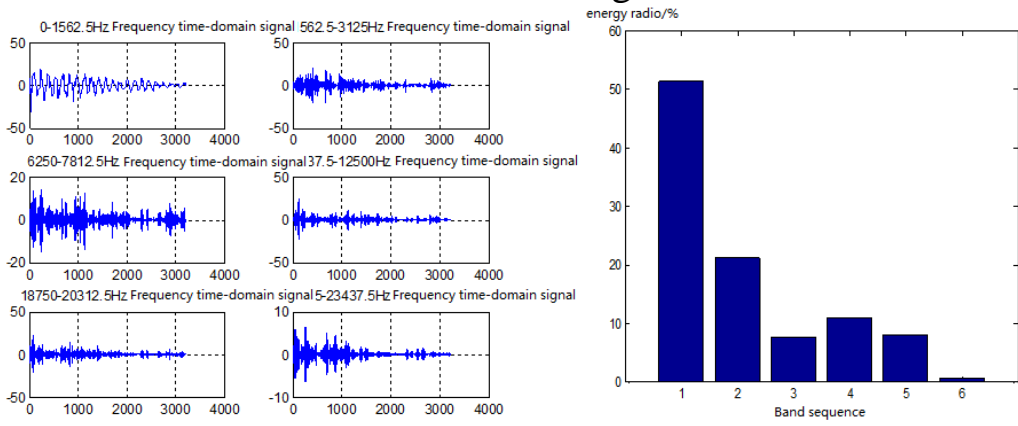

Fig. 8 the 2nd Shell Recoil Progress Vibration Signal each Band Time-domain and Energy Distribution Diagram
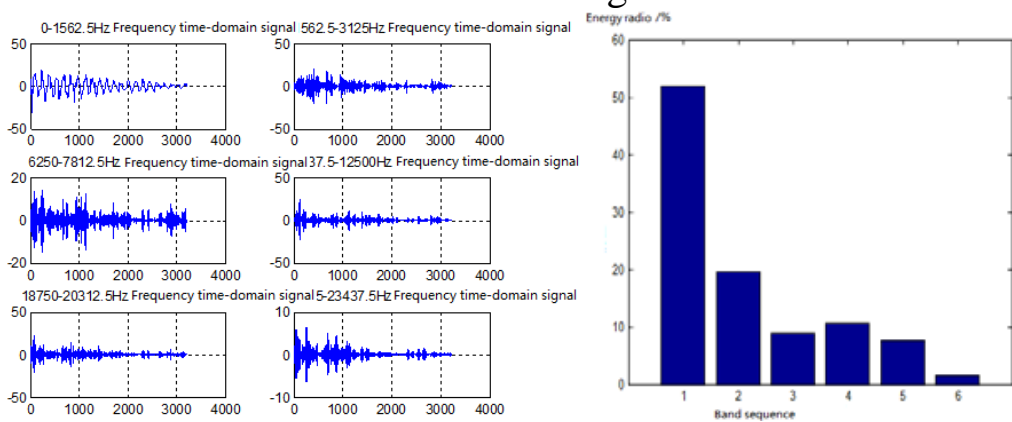

Fig. 9 the 3rd Shell Recoil Progress Vibration Signal each Band Time-domain and Energy Distribution Diagram 


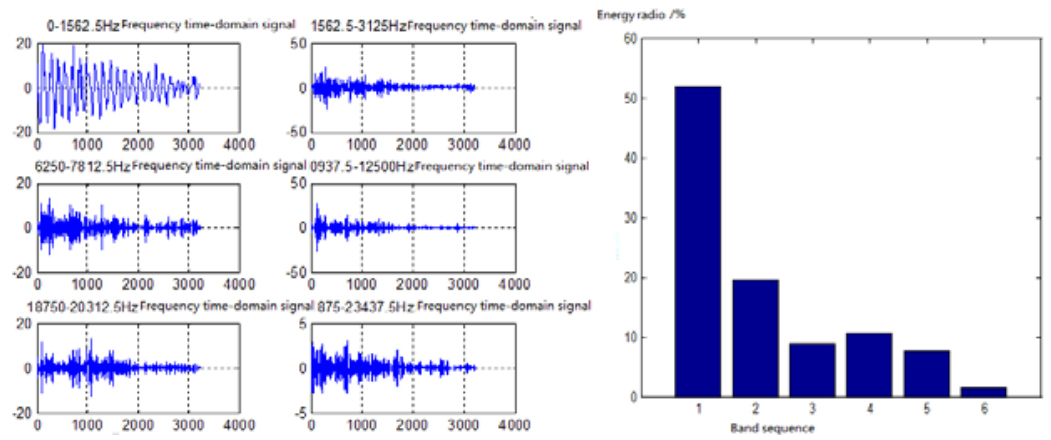

Fig. 10 the 4th Shell Recoil Progress Vibration Signal each Band Time-domain and Energy Distribution Diagram

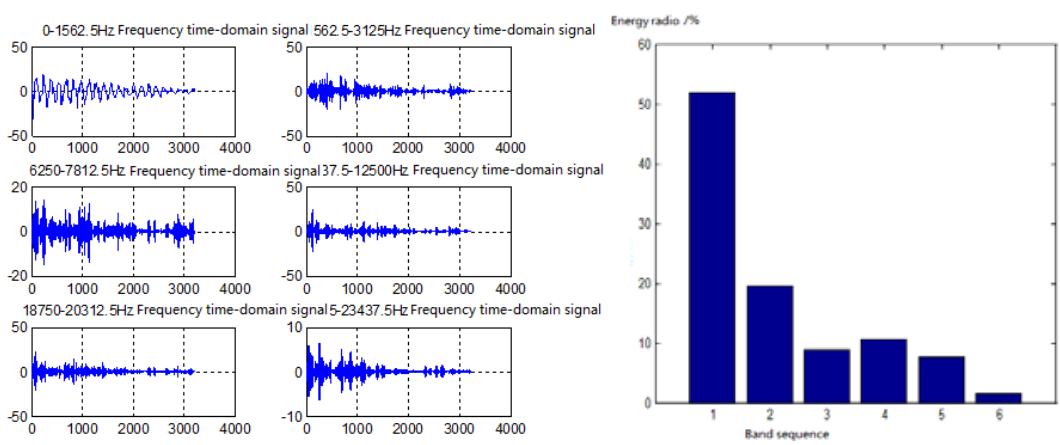

Fig. 11 the 5th Shell Recoil Progress Vibration Signal each Band Time-domain and Energy Distribution Diagram

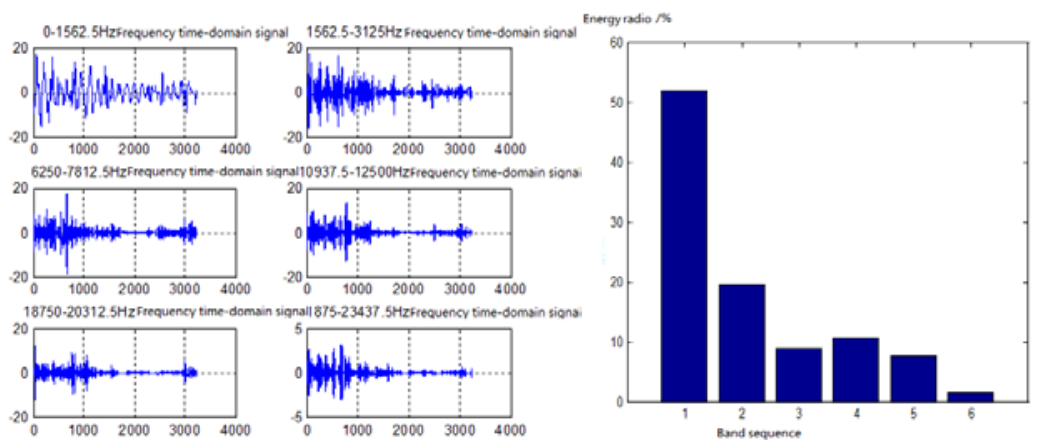

Fig. 12 the 6th Shell Recoil Progress Vibration Signal each Band Time-domain and Energy Distribution Diagram

Table 1 Actual Test of Vibration Signal Wavelet Packet Decompose Extract Energy Eigenvector

\begin{tabular}{|c|c|c|c|c|c|c|}
\hline $\begin{array}{c}\text { Band } \\
\text { Sequence }\end{array}$ & $\begin{array}{c}0 \\
- \\
1562.5 \\
\mathrm{~Hz}\end{array}$ & $\begin{array}{c}1562.5 \\
- \\
3125 \\
\mathrm{~Hz}\end{array}$ & $\begin{array}{l}6250 \\
\quad- \\
7812.5 \\
\mathrm{~Hz}\end{array}$ & $\begin{array}{c}10937.5 \\
- \\
12500 \\
\mathrm{~Hz}\end{array}$ & $\begin{array}{c}18750 \\
- \\
20312.5 \\
\mathrm{~Hz}\end{array}$ & $\begin{array}{c}21875 \\
- \\
23437.5 \\
\mathrm{~Hz}\end{array}$ \\
\hline 1 & 52 & 20.30 & 7.69 & 11.71 & 7.18 & 0.81 \\
\hline 2 & 51.40 & 21.21 & 7.62 & 11.01 & 8.02 & 0.70 \\
\hline 3 & 51.82 & 19.50 & 8.88 & 10.60 & 7.71 & 1.49 \\
\hline 4 & 53.91 & 17.76 & 8.31 & 10.26 & 7.19 & 2.12 \\
\hline 5 & 52.54 & 18.76 & 8.58 & 10.68 & 7.14 & 2.31 \\
\hline 6 & 51.43 & 18.81 & 9.54 & 10.08 & 7.93 & 2.21 \\
\hline
\end{tabular}

Table 1 further calculated from the characteristic values obtained for each issue that is high-frequency energy ratio were 19.70\%, 19.73\%, 19.80\%, 20.02\%, 20.13\%, 20.22\%.

\section{Recoil vibration signal characteristic analysis}

Table 1 feature vectors can draw the following conclusions

(1)Recoil low frequency vibration signal energy means decreasing significantly, decreasing the high frequency energy is not obvious. 
(2)Recoil vibrations caused by the movement itself mainly in the high frequency vibration signal.

(3)Wear rings and other components of control leads to high frequency vibration signal energy increases.

Detailed analysis of the quantitative relationship between the amount of wear and features below. The relation of control ring wear and the maximum recoil displacement between the virtual prototype technologies, as shown in Figure 13[5]. Based on the test while recoil system vibration signal also collected recoil system displacement signal, so you can build relationships and maximum recoil displacement between the proportions of high-frequency energy, as shown in Figure 14. In conjunction with Figure 13 and 14, further relations Recoil volume control ring wear and recoil system vibration signal between the high frequency energy than, as shown in Figure 15.

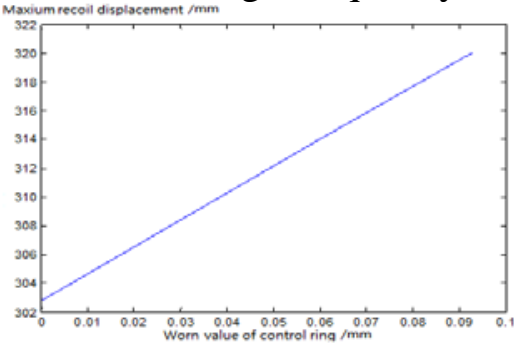

Fig. 13 the Relation of Maximum Recoil Displacement and Worn Value of Control ring

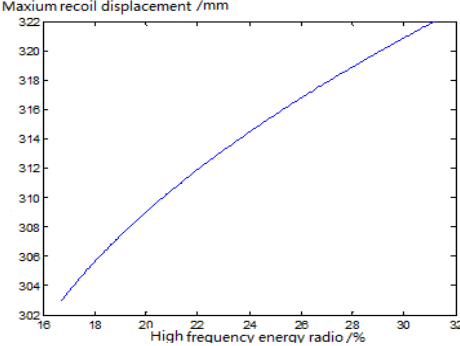

Fig. 14 the Relation of Maximum Recoil Displacement and HF Energy Radio

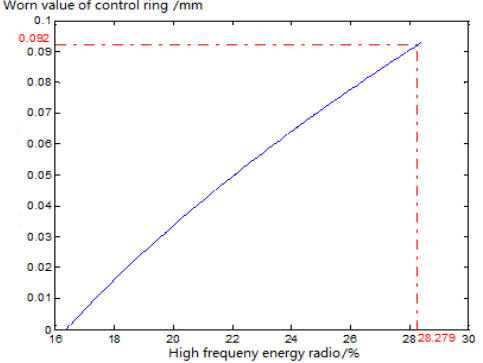

Fig. 15 the Relation of Worn Value of Control Ring and Worn Value of Control Ring

Figure 15 shows that moderate recoil system of ring wear limit of approximately $0.092 \mathrm{~mm}$, and when the proportion of $28.279 \%$ high-frequency energy, control ring wear reaches this value, recoil system in a very unstable state, prone to big the failure, resulting in a large loss of property personnel. Therefore, when monitored recoil system vibration signal high-frequency energy than close to this value, we must stop firing artillery, anti-recoil means timely maintenance. The control ring wear model and artillery projectiles made several combined life prediction model can also be set up recoil system, and make predictions of artillery fire mission success.

\section{Summary}

In this paper, wavelet packet method of firing the vibration signals measured by the recoil means extract features. Finally, according to the wavelet packet energy feature vectors extracted feature amount to establish a relationship between the control ring wear recoil system, laid the foundation for the recoil device failure prediction and diagnosis.

\section{Acknowledgement}

In this paper, the research was sponsored by the Nature Science Foundation of Academy of Armored Force Engineering (Project No. 2011CJ052). 


\section{References}

[1] Han Zhifeng,Song Zhihuan,Bearing Noise Analysis and Fault Monitoring Wavelet Transform.[J] University of Science and Technology of China based.2005, (35): 186-191.

[2] Han Zhennan, Li Jinbao, Xiong, Shibo Wavelet Neural Network in gear early fault diagnosis [J]. Mining Machinery. 2003(5): 89-90.

[3] Zheng Haibo.Non-stationary non-Gaussian signal feature extraction and fault diagnosis technology research [D] Hefei: Hefei University Press, 2003.

[4] Hu Qing, Han Xiaoming, Emulsion Pump Crankshaft Bearing Wear Failure Diagnosis Based on the Band Energy Analysis [J] Mining Machinery 2008, 36 (17): 19-22.

[5] Zhang Jingbo, Cheng Li, Hu Huibin. Based on Fault Simulation Technology [J] take virtual prototyping inverted Computer Measurement \& Control 2012, 20 (5): 1287-1289. 